\title{
Research on Moving Target Tracking Based on Kalman Filter in Volleyball Video
}

\author{
Zhiyong $\mathrm{Xu}^{1}$ and Shangbin $\mathrm{Li}^{2 *}$ \\ ${ }^{1}$ Jiangsu Vocational Institute of Commerce, Nanjing 211168, China, \\ ${ }^{2}$ Physical Education Department, Harbin Engineering University, Harbin 150001, \\ China, \\ ${ }^{1}$ xuzhiyong2050@sina.com and ${ }^{2}$ lishangbin@hrbeu.edu.cn
}

\begin{abstract}
Sport videos are becoming more and more popular. Therefore, it has highly practical significance and commercial value to analysis sport videos. We researched on the moving object detection, prediction tracking and trajectory in volleyball video sequences captured by a fixed camera. The objects include players and volleyball. For moving objects tracking, firstly, the ID and location information of the objects blob are received through image segmentation. Secondly, tracking algorithm is a combination of centroid tracking algorithm and Kalman filter. The algorithm can provide players and volleyball object tracking. In the process of tracking, Kalman filter is imposed to forecast the motion information to improve the tracking precision.
\end{abstract}

Keywords: Sports Video, Moving object detection, Tracking, Background subtraction

\section{Introduction of Traditional Tracking Algorithms}

Moving target tracking is a problem of corresponding matching built according to features such as target shape [1-10], color and texture between consecutive images. It aims to get the location coordinate of moving target in each frame image as thus to conclude the target's motion trail through data association [11-18].

Recently, owing to researchers' great endeavors, plenty of target tracking methods have been proposed [19-21]. In terms of the principle, such method includes four types: modelbased tracking, contour-based tracking, region-based tracking and feature-based tracking [22-23].

\subsection{Model-Based Tracking Methods}

In the model-based tracking method, different models are constructed in accordance to structure feature of tracked target; then the target is traced in subsequent image sequence. The method is simple and easily implemented. In general there are three kinds of model representation way:

(1) linear graphic methodology- it approximately represents every part of moving target in the form of straight line; in [24], Karaulova et al., established the hierarchical model of kinesiology for trailing human body in video sequence;

(2) contour model-this method projects tracked target to image, then follows it up as per position change in the image; in [25], Ju et al., presented paperboard human model; human body is represented by a set of connecting planar area blobs; the parametric movement of those blobs are restricted by joint motion; the model was used to analyze joint movement images;

(3) three-dimensional Model in the model, structural details of moving target is

${ }^{*}$ Corresponding Author 
described by means of three dimensional model like generalized elliptic cylinder, ball and frustum; it involves huge computation; in [26], Zhu et al., traced vehicles with cuboidshaped car model in VISATRAM system to acquire target's speed and size; in [27], Nickels et al., realized motion tracking of manipulator in the stereoscopic space.

Experimental findings reveal that the accuracy of model-based tracking method depends on the means for constructing target and it can achieve better tracking effect; for non-rigid objects, the method can implement tracking in complicated environment through model design; however it requires abundant computation; also because of bad instantaneity, it can hardly realize tracking of multiple targets.

\subsection{The Contour-Based Tracking}

The contour-based tracking utilizes closed curve outlines to depict moving object and the contour can renew automatically. This method fetches the shape of target and provides more effective description of target. In [28], Deriche applied Geodesic active contour to detect and tail after several targets in the image sequence in the combination with Level Set theory. In [29], Yokoyama et al., extracted target edges with optical flow method; then chased target with background subtraction method. The object finally appeared in the form of approximate contour.

The experiment proves that contour-based tracking method requires less computation; if every moving target can be reasonably discerned and contour initialization is achieved, the method can track target consecutively even if the target is partially covered; however in most cases, it's quite difficult to initialize.

\subsection{The Region-Based Tracking}

The region-based tracking method regards the area where target locates as research goal; it fetches some features of that area as to chase the target. In [30], Schmaltz et al., developed region-based gesture tracking algorithm. The technique worked well for rigid objects; but when moving target was covered or shadow appeared, the algorithm performed badly. Region-based tracking method together with color model overcomes incomplete shelter of the target and improves target tracking accuracy.

The test demonstrates that the pure region-based target tracking algorithm is too sensitive to the problem of target shadow and shelter. If better tracking effect is required, we can combine color information and gradient information to create adaptive background model, with the use of background subtraction method to eliminate influences of shades as to extract moving area.

\subsection{The Feature-Based Tracking}

The feature-based tracking is implemented with the help of moving target's features which can differentiate foreground and background area in the target tracking process. This kind of tracking includes two steps: target's feature extraction and feature matching.

Feature extraction aims to obtain independent and distinguishable features of every target image. The selection of feature should have relative stability and divisibility in line with the necessary characteristic quantity chosen for target recognition and tracking. In the premise of guaranteeing recognition precision and reliability, it's better to choose possibly fewer feature numbers. In [31], Jang and Choi completed tracking of moving target with feature information like regional shape, texture and color, the robustness of tracking being enhanced. Feature matching [32] uses feature which can help discriminate moving target as benchmark; then search moving target which needs tracking from video sequence waiting for matching as to determine the position of target in the image.

\section{Proposed Moving Target Tracking Algorithm}




\subsection{Features of Moving Target}

Features of moving target provide important basis for target tracking. In plentiful tracking algorithms, target feature extraction is an essential step. The extracted target features can still describe well moving target even when changes happen to the target itself, environment or movement. Image target generally has the following four types of features:

1. Image's visual feature like image boundaries, contour, Shape, texture and other features;

2. Image's statistical feature like color histogram, various invariant moments;

3. Image conversion coefficient feature such as wavelet transform coefficient, auto-regressive model feature;

4. Image's algebraic feature e.g., singular value decomposition of image matrix.

Here we feature the centroid of moving target and use it as feature value of target tracking. In the binarized image of foreground moving target and background image segmentation, set grey value $G(x, y)$ of one pixel ; $(x, y)$ image size $M \times N$; then target's centroid coordinate $\left(x_{0}, y_{0}\right)$ is:

$$
x_{0}=\frac{\sum_{i=0}^{M-1} \sum_{j=0}^{N-1} x_{i, j} G(i, j)}{\sum_{i=0}^{M-1} \sum_{j=0}^{N-1} G(i, j)}, y_{0}=\frac{\sum_{i=0}^{M-1} \sum_{j=0}^{N-1} y_{i, j} G(i, j)}{\sum_{i=0}^{M-1} \sum_{j=0}^{N-1} G(i, j)}
$$

\subsection{Introduction of Kalman Filter}

When background environment is complicated, we use centroid tracking algorithm to trail moving target. In one-frame or multi-frame image, for many reasons, target would diverge from the normal track or even disappear, which is not favorable for target tracking in current frame and thereafter. To address the headache, we introduce Kalman filtering method. Based on the last one and current frame, it can predict tracking result, keeping better tracking effect.

Kalman filtering method was firstly proposed by Kalman and Bucy in 1960 to solve the problem of discrete data linear filter. As an optimal recursive filtering method, it was initially applied in aerospace industry before extensive applications in many other fields.

Kalman filtering keeps to the basic thought: iterate constantly the time sequence at forecast and update stage to diminish errors as a result of system noise and observation noise to get the best status parameters. Instead of saving historical measured data, Kalman filter takes advantage of its own state transition equation to figure out new parametric estimations through a set of recursive formula, reducing storage capacity and calculated amount.

Suppose target's movement is linear and location signal noise is Gaussian white noise. It predicts tracking through two phases:

1. Prediction stage: includes prediction of system state vector and system error covariance matrix;

2. Correction stage: correct the predictive vector of system state with system measurement formula to get optimal system state value. Its working process is shown in Figure 1. 


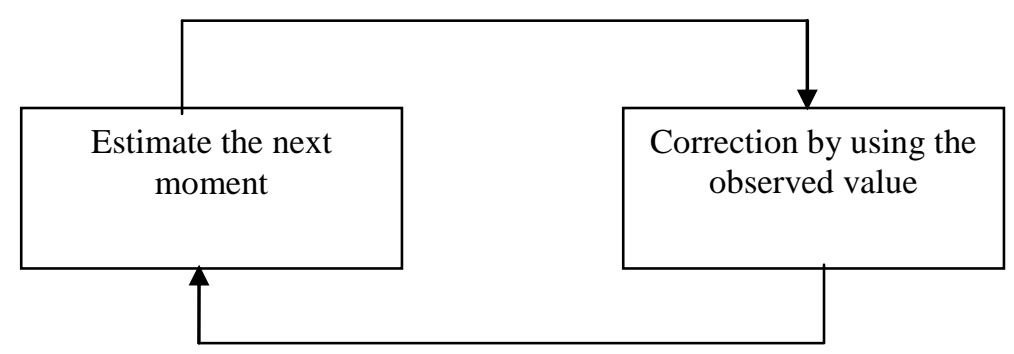

Figure 1. The Working Process of Kalman Filter

A discrete dynamic system is described as follows:

Equation of state prediction:

$x_{k}=A_{k} x_{k-1}+w_{k-1}$

Equation of state prediction:

$$
y_{k}=C_{k} x_{k}+v_{k}
$$

Where, Matrix $A_{k}$ and $C_{k}$ are respectively the state transition matrix and observation matrix of the system, $w_{k-1}$ and $v_{k}$ are system noise.

The estimation problem arises because the noise of $w_{k-1}$ and $v_{k}$, if not to consider the noise by the Formula (1) and (2) can be obtained by $\hat{x}_{k}^{\prime}$ and $\hat{y}_{k}^{\prime}$, there are:

$$
\begin{aligned}
& \hat{x}_{k}^{\prime}=A_{k} \hat{x}_{k-1}^{\prime} \\
& \hat{y}_{k}^{\prime}=C_{k} A_{k} C_{k-1}^{-1} \hat{y}_{k-1}^{\prime}
\end{aligned}
$$

Due to ignoring the noise of $w_{k-1}$ and $v_{k}, \hat{y}_{k}^{\prime}$ deviation from the $y_{k}$ error, the error is expressed by $\tilde{y}_{k}$, then

$$
\tilde{y}_{k}=y_{k}-\hat{y}_{k}^{\prime}
$$

If the $\tilde{y}_{k}$ is multiplied by an error matrix $H_{k}$ to modify the original $\hat{x}_{k}^{\prime}$, the result is optimal linear estimate of $x_{k} . P_{k}$ shows the average variance, after pushing :

Mean square deviation prediction equation:

$P_{k}^{\prime}=A_{k} P_{k-1} A_{k}^{T}+Q_{k-1}$

Kalman gain equation:

$$
H_{k}=P_{k}^{\prime} H_{k}^{T}\left(H_{k} P_{k}^{\prime} H_{k}^{T}+R_{k}\right)^{-1}
$$

State correction equation:

$\hat{x}_{k}=A_{k} x_{k-1}^{\prime}+H_{k}\left(y_{k}-C_{k} A_{k} x_{k-1}\right)$

Mean square error correction equation:

$P_{k}=\left(I-H_{k} C_{k}\right) P_{k}^{\prime}$ 


\subsection{Blob Tracking Algorithm Combining Centroid Tracking and Kalman Filter}

The key to moving target tracking is to perform feature matching between the detected foreground target and tracked moving target. The paper employs the combined method of centroid tracking and Kalman filter to chase motion target. First of all, we calculate feature value with the use of result in above part; then build motion model with Kalman filter to predict the current target to quicken the speed of target matching; lastly, update motion model to have movement trajectory of moving target.

In order to track multiple targets in videos, we introduce the concept of blobs. We create a blob tracking table. In the next one frame, we do matching of every blob's feature information, which helps increase the accuracy of tracking multiple targets.

2.3.1. The Blob-Based Tracking: The blob-based tracking algorithm has fundamental principles: make image segmentation of candidate pixel; judge the pixel belongs to target or background or other region.

Here we applied blob-based idea to do tracking of detected objects. According to characteristics of volleyball videos, we design blob tracking into two modules: new blob detection and blob tracking.

New blob detection module is responsible to detect blobs in foreground mask codes. , the module structure diagram is shown in Figure 2.

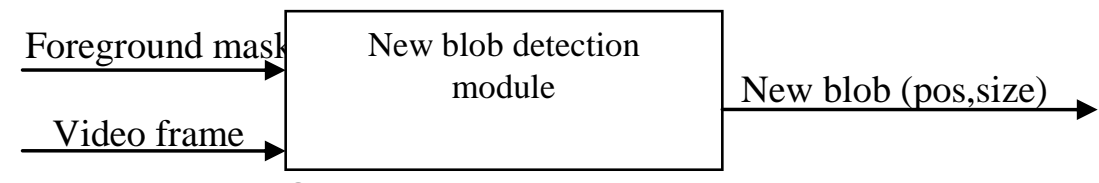

Figure 2. The Structure of New Blob Detection Model

Blob tracking module uses result got by new blob detection module to initialize it and track new incoming blobs. Its module structure is shown in Figure 3.

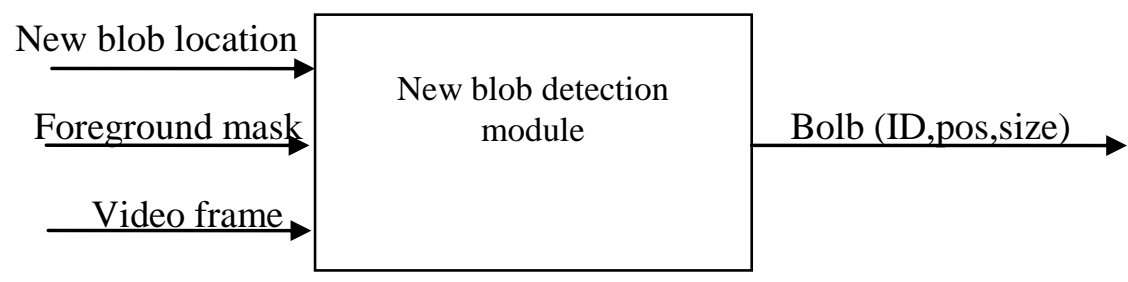

Figure 3. The Structure of Blob Tracking Model

New detected blobs are put in blob tracking module table, in order to simplify the creation of blob tracking module. The specific mass tracking list schematic diagram is shown in Figure 4. 


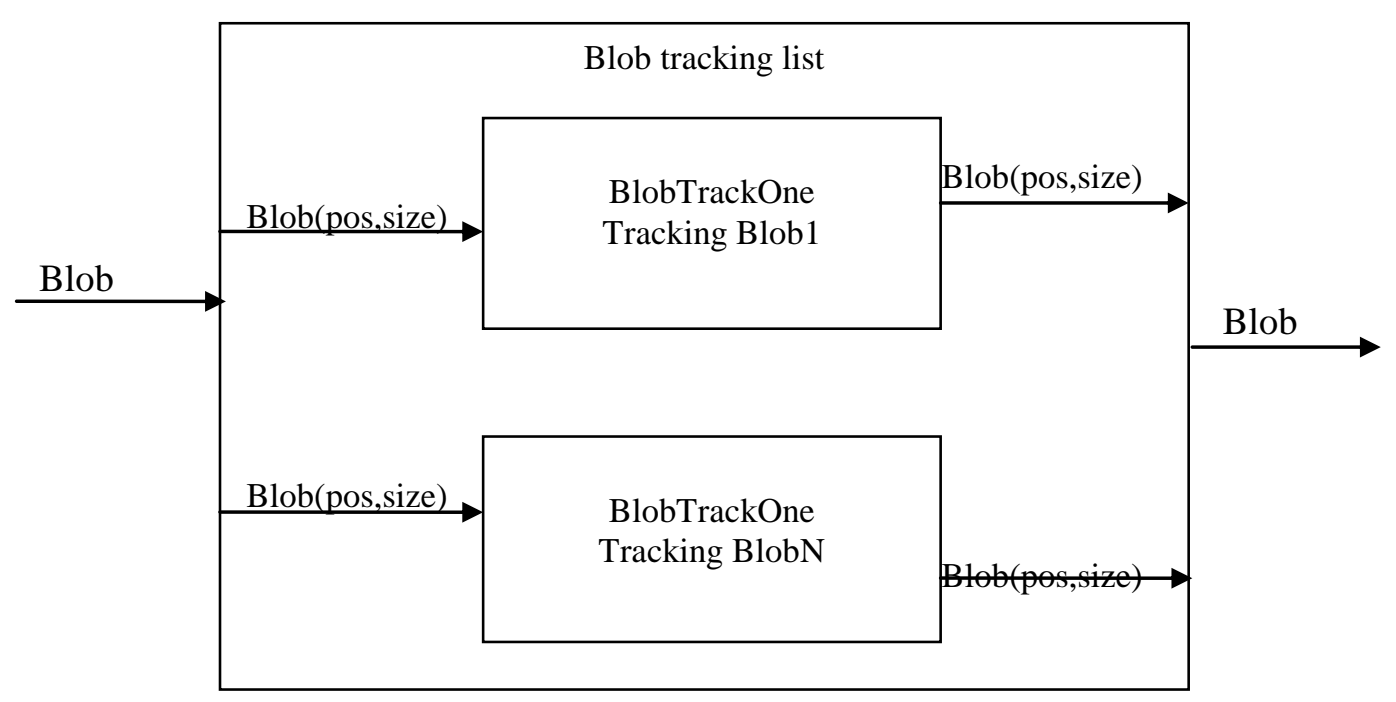

Figure 4. The List Diagram of Blob Tracking

2.3.2. Kalman Tracking Model: Kalman tracking model can be divided into four modules:

(1) Feature value calculation: calculate moving target's feature value, center of mass and position size with extracted results;

(2) Movement model: use Kalman filter to build system motion model, define state vector and foresee the position where moving target possibly appears in the next one frame;

(3) Feature matching: define target's similar function, calculate value of similar function with feature value, judge if it's identical moving target;

(4) Model update: use new motion model as input of the next motion model.

Based on Kalman filter, we set state vector $s_{k+1}$ at time moment $\mathrm{k}+1$ in the model, which is determined by vector $s_{k}$ at point $\mathrm{k}$, conversion function $\mathrm{A}$ and noise $w_{k}$; observation vector $z_{k+1}$ is decided by vector $s_{k+1}$ at point $\mathrm{k}+1$, observation function $\mathrm{C}$ and noise $v_{k+1}$.

Equation of state prediction:

$s_{k+1}=A s_{k}+w_{k}$

Equation of state prediction:

$z_{k+1}=C s_{k+1}+v_{k+1}$

Where, $w_{k}$ and $v_{k+1}$ are the noise, $s_{k}$ is the state vector, $z_{k+1}$ is the observation vector, the vector composition, such as the Formula (12) is shown:

$s_{k}=\left[x_{k}, y_{k}, \dot{x}_{k}, \dot{y}_{k}, L_{x k}, L_{y k}, \dot{L}_{x k}, \dot{L}_{y k}\right]^{T}$

$z_{k+1}=\left[x_{k+1}, y_{k+1}, L_{x k+1}, L_{y k+1}\right]^{T}$

Set sampling time $t$ is very short, can be similar to that of the target to move at a uniform speed, and the window does not change, then the state transition matrix A is: 


$$
A=\left[\begin{array}{llllllll}
1 & 0 & t & 0 & 0 & 0 & 0 & 0 \\
0 & 1 & 0 & t & 0 & 0 & 0 & 0 \\
0 & 0 & 1 & 0 & t & 0 & 0 & 0 \\
0 & 0 & 0 & 1 & 0 & t & 0 & 0 \\
0 & 0 & 0 & 0 & 1 & 0 & t & 0 \\
0 & 0 & 0 & 0 & 0 & 1 & 0 & t \\
0 & 0 & 0 & 0 & 0 & 0 & 1 & 0 \\
0 & 0 & 0 & 0 & 0 & 0 & 0 & 1
\end{array}\right]
$$

Observation matrix $\mathrm{C}$ is:

$$
C=\left[\begin{array}{llllllll}
1 & 0 & 0 & 0 & 0 & 0 & 0 & 0 \\
0 & 1 & 0 & 0 & 0 & 0 & 0 & 0 \\
0 & 0 & 1 & 0 & 0 & 0 & 0 & 0 \\
0 & 0 & 0 & 1 & 0 & 0 & 0 & 0
\end{array}\right]
$$

After state equation and observation equation of motion model are defined, we use Kalman filter to estimate the position and window size of target's centroid, narrowing down the scope of searching centroid of next one frame.

2.3.3. Target Feature Matching: Feature matching refers to comparing segmented targets in different frames with their features. Here we use target's centroid distance and contour area as target feature to do matching. If matching succeeds, it's old blob; otherwise it's called new blob, which is put in blob tracking table.

A group of moving image sequence is got in the interval $\mathrm{t}$, denoted:

$$
F=\left\{f_{1}, f_{2} \ldots f_{k}\right\}
$$

If the $\mathrm{k}$ frame image $\mathrm{m}$ has a $f_{k}$ target, the centroid of the ith target and the window width can be recorded as $x_{k}^{i}, y_{k}^{i}, L_{x k}^{i}, L_{y k}^{i}$.

The formula for the distance function and the window area function defined by the centroid of the $\mathrm{i}$ target of the $\mathrm{k}$ frame and the $\mathrm{j}$ target of $\mathrm{k}+1$ frame are respectively.

$$
\begin{aligned}
& D(i, j)=\frac{\left|c_{k}^{i} c_{k+1}^{j}\right|}{\max \left|c_{k}^{i} c_{k+1}^{j}\right|} \\
& A(i, j)=\frac{\left|a_{k}^{i}-a_{k+1}^{j}\right|}{\max \left|a_{k}^{i}-a_{k+1}^{j}\right|}
\end{aligned}
$$

Where, $c_{k}^{i} c_{k+1}^{j}=\sqrt{\left(x_{k}^{i}-x_{k+1}^{j}\right)^{2}+\left(y_{k}^{i}-y_{k+1}^{j}\right)^{2}}, a_{k}^{i}-a_{k+1}^{j}=L_{x k}^{i}, L_{y k}^{i}-L_{x k+1}^{j} L_{y k+1}^{j}$

$$
D(i, j) \leq 1 \text { and } A(i, j) \leq 1 \text {. }
$$

Similar function is:

$\delta(i, j)=\xi D(i, j)+\zeta A(i, j)$

Where, $\xi>\zeta, \xi+\zeta=1, \delta(i, j) \leq 1$. 
2.3.4. Model Updating Design: When similar function minimum is found, it means the trailing of the same moving target is found; at this moment, use feature value of the jth moving target in the $(\mathrm{k}+1)$ th frame as input for motion model to estimate next frame. Repeat iterations to complete model update.

During target tracking, we use target location and predicted location by Kalman filter for target matching. Once matching succeeds, use new feature value to update Kalman filtering model. Write down centroid position of moving target in each frame; incorporate them to target's motion trajectory; repeat the process till the tracking process of moving target finishes.

In the algorithm here, moving target tracking method and extraction method are actually supplementary to each other. On the one hand, target extraction is separate, not dependent on the predicted position by tracking filter; even though the filter has deviations, target detection accuracy won't be impacted; with detection result, we can constantly adjust and renew filter parameters to make it better to track and predict target; on the other hand, after tracking filter catches well the movement rule of target, it can correct detection results to make the method more reliable.

\section{Experimental Analysis and Results}

Considering features of moving target in volleyball videos, we carry out experiments to validate the proposed blob tracking algorithm which combines Kalman filtering with different videos. Figure 5, is testing on tracking of volleyball players; Figure 6, is testing on tracking of volleyball; video sequence is avi format, captured by fixed camera at $25 \mathrm{fps}$. The system has two sub windows, displaying separately original video and tracking result of moving target.

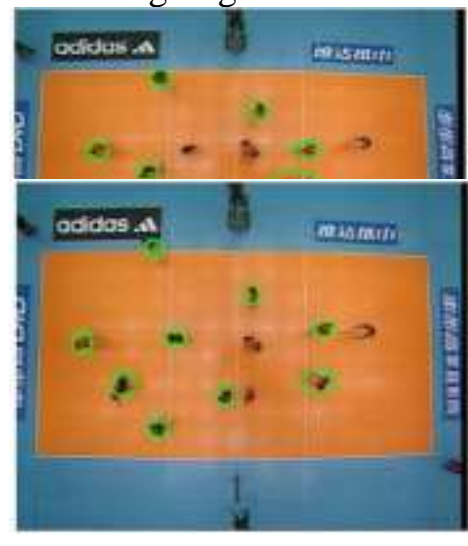

62th frames

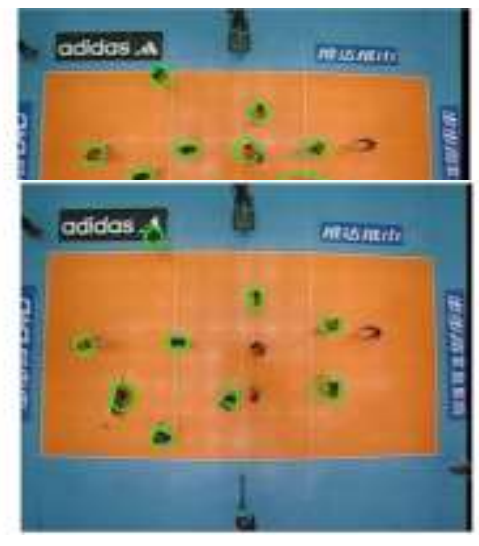

72th frames

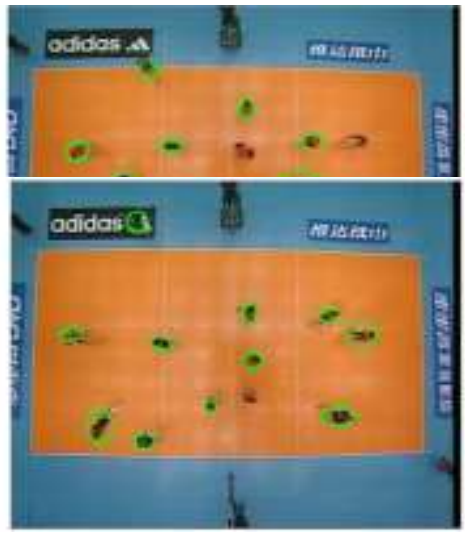

109th frames

\section{Figure 5. Player Tracking}

We chose volleyball videos by camera fixed on the top to do player tracking test. Figure 5, is effect picture of player tracking. From the experiment, we see since camera is fixed on the top, no shelters exist among players; player tracking result is very stable; player's tracking area varies from time to time along with their movements. In the whole tracking course, the algorithm maintains favorable accuracy and stability.
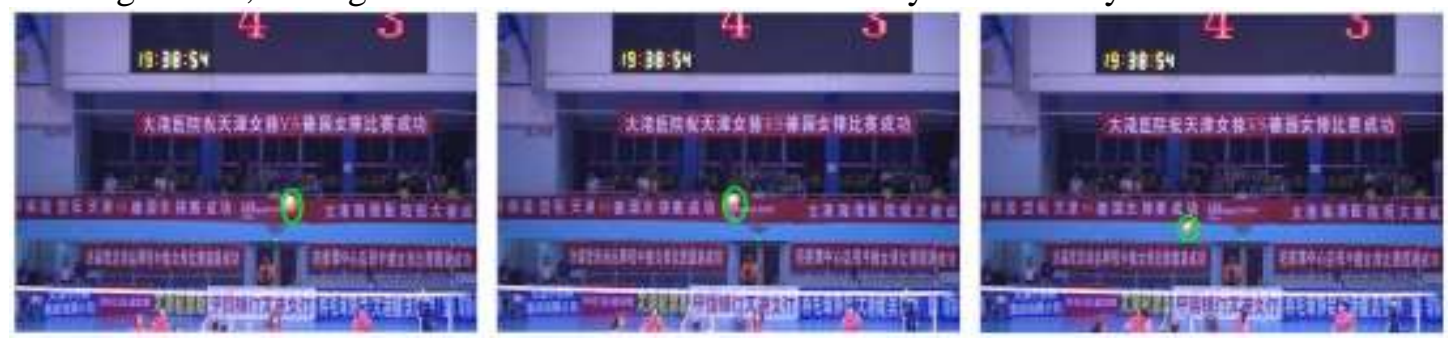


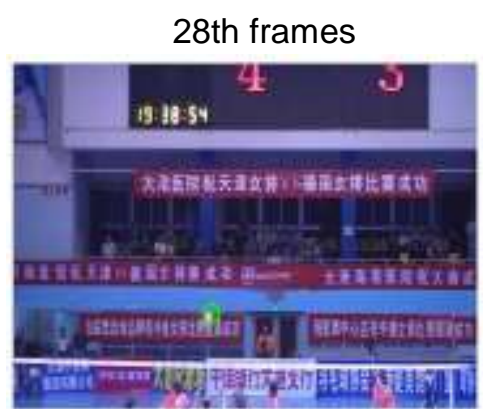

40th frames

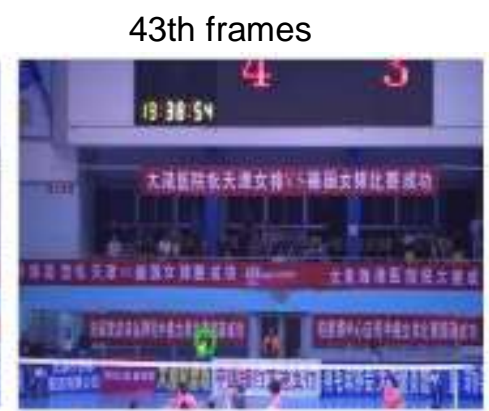

43th frames

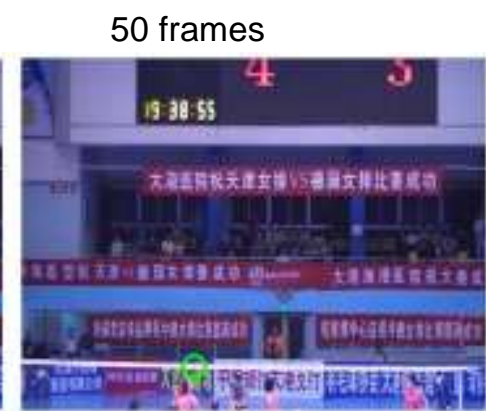

50thframes

\section{Figure 6. Volleyball Tracking}

We chose volleyball videos by camera fixed at one side of the pitch to do volleyball tracking test. Figure 6, is effect picture of player tracking. The experiment suggests that by choosing shape-based features for volleyball tracking, it avoids interference of complicated scenes with volleyball tracking, able to track the ball accurately; the tracking area basically keeps unchanged. When tracked target is covered by other moving target, it will get lost. Thanks to the use of Kalman filter for motion estimation, by predicting the position of volleyball in next frame, the algorithm is able to find back lost target as soon as possible. The algorithm efficiency is improved.

It can be seen from the above experimental results that the algorithm is still able to track moving targets in complex scenes, and has high accuracy and robustness.

\section{Conclusion}

In this paper, we first introduce some traditional target tracking methods, and then compare the principle and characteristics of the algorithm, and then introduce the feature of the moving object and the Kalman filter. Based on the proposed tracking method in the paper, namely centroid tracking algorithm combined with Kalman filter of Block tracking algorithm. Experiments show that the algorithm is good tracking results, using Kalman filter estimation can improve tracking accuracy.

\section{References}

[1] W. Weihua, "The method of detection and behavior recognition in Intelligent Visual Surveillance moving target”, Xi'an Electronic and Science University, (2014).

[2] Y. Lei, "Study of object detection and tracking in traffic video detection system", Chang'an University, (2010).

[3] Z. Zhixu, "The moving target detection and tracking in intelligent visual surveillance", University of Electronic Science and technology, (2009).

[4] Y. Huaxin, "Remote sensing image target detection and target tracking based on motion", Xi'an Electronic and Science University, (2014).

[5] Z. Weili, "Study on target tracking based on monocular vision", University of Electronic Science and technology, (2013).

[6] Claire, "Research on moving target tracking system based on vision", Wuhan University of Science and Technology, (2012).

[7] D. Feng, "Software design of intelligent video surveillance system based on DVR", University of Electronic Science and technology, (2012).

[8] F. L. Jing, "Using a moving object tracking algorithm based on Calman filter", Application Research of computers, vol. 08, (2010), pp. 3162-3164.

[9] L. Lijuan and C. Jianchun, "Detection and tracking of moving objects in video sequences", Electronic Science and technology, vol. 09, (2010), pp. 92-95.

[10] Xu Hanin, time course, Hou Honglu, Li politically correct. Based on a simplified UKF interacting multiple model video image tracking algorithm for maneuvering target. Opto electronic engineering, (2010), vol. 11, pp. 14-18. 
[11] L. Xining, Y. Gang, Y. Xuecai, W. Shiyang, Z. Liang, S. Ke and C. Tao, "A dynamic template matching method of Calman filter tracking”, Photoelectric engineering, vol. 10, (2010), pp. 29-33.

[12] X. J. Zhao, "An improved moving target anti occlusion tracking algorithm", Journal of Beijing University of Aeronautics and Astronautics, vol. 04, (2013), pp. 517-520.

[13] W. Jian, Z. Lei and L. Meng, "The realization of the moving target tracking based on Calman filter", Silicon Valley, vol. 10, (2013), pp. 62-73.

[14] Q. Shiru and Y. Honghong, "Multi target detection and tracking of video sequences using Kalman_BP neural network ", infrared and laser engineering, vol. 09, (2013), pp. 2553-2560.

[15] Z. Naijiang and Li. Chengdong, "The target tracking method of coal preparation plant personnel based on improved MeanShift algorithm", automation of mining and mining, vol. 02, (2012), pp. 32-35.

[16] W. Huilan, L. Hangfeiaand L. long, "Matlab for real-time tracking of moving object detection system ", computer security based on vol. 03, (2012), pp. 17-20.

[17] Q. Hongbiao and L. Wei, "A new nonlinear filter of multi feature fusion tracking algorithm", Application Research of computers, vol. 05, (2012), pp. 1737-1740.

[18] H. Shi, M. Xue, Z. Jian and Z.Jifa, "Based on the level set method of weak boundary moving human target tracking and contour extraction", Computer science, vol. 05, (2012), pp. 239-242.

[19] Y. Ping and L. Xiaorun, "Based on the video detection technology of the smart tunnel traffic safety monitoring system design", Computer applications and software, vol. 06, (2012), pp. 128-130.

[20] J. G. Chen, R. Bingqing and M. Lili, "gentle and quiet. Under the condition of unknown noise variances of video target tracking", China Journal of image and graphics, vol. 07, (2015), pp. 906-913.

[21] Z. Weili, J. Chunhua and Ha.Zongbo, "Target tracking algorithm based on improved mean shift and Calman filter", computer applications and software, vol. 05, (2014), pp. 240-243.

[22] Q. Yiping and Y. Daoye, "Journal of algorithms and behavior analysis", Beijing University of technology tracking based on Kalman filter vehicle video detection and vol. 07, (2014), pp. 1110-1113.

[23] S. Shaojun, L. Hui and S. Huajun, "A compression tracking algorithm based on Calman filter", Shandong science, vol. 05, (2014), pp. 54-59.

[24] I. Karaulova, P. Hall and A. Marshall, "A Hierarchical Model of Dynamics for Tracking People with a Single Video Camera”, Proc. British Machine Vision Conference, vol. 9, (2000), pp. 352-361.

[25] S. Ju, M. Black and Y. Yaccob, "Cardboard People: A Parameterized Model of Articulated Image Motion”, Proc. IEEE International Conference on Automatic Face and Gesture Recognition, vol. 10, (1996), pp. 38-44

[26] Z. Zhigang, X. Guangyou and Y. Bo, "VISATRAM:A real-time vision system for automatic traffic monitoring", Image and vision Computing, vol. 18, no. 10, (2000), pp. 781-794.

[27] K. Nickels and S. Hutchinson, "Estimating uncertainty in SSD-based feature tracking", Image and Vision Computing, vol. 20, no. 1, (2002), pp. 47-58.

[28] Y. Zhong, A. Jain and M. Dubuisson-Jolly, "Object tracking using deformable templates", IEEE transactions Pattern Analysis and Machine Inlelligence, vol. 22, no. 5, (2000), pp. 544-549.

[29] M. Yokoyama and T. Poggio, "A Contour-Based Moving Object Detection and Tracking”, IEEE International Workshop on Visual Surveillance and Performance Evaluation of Tracking and Surveillance Processing, vol. 10, (2005), pp. 271-276.

[30] C. Schmaltz, B. Rosenhahn and T. Brox, "Region-Based Pose Tracking", Pattern Recognition and Image Analysis Processing, vol. 6, (2007), pp. 56-63.

[31] D. S. Jang and H. I. Choi, "Active models for tracking moving object", Pattern Recognition, vol. 33, no. 7, (2003), pp. 1135- 1146.

[32] W. Weihua, L. Cuihua and S. Hua, "Moving object detection technology in video sequences", Engineering Journal, vol. 27, no. 3, (2006), pp. 446-450.

\section{Authors}

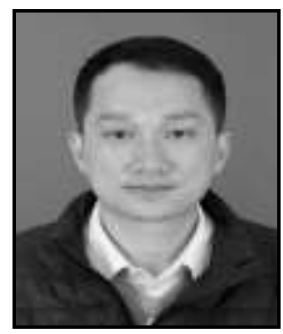

Zhiyong Xu, He received his B.S and M.S degree from Nanjing Institute of Physical Education and Sports. He is a lecturer at Jiangsu Vocational Institute of Commerce. He is in the research of physical education; movement training; management of sport. 